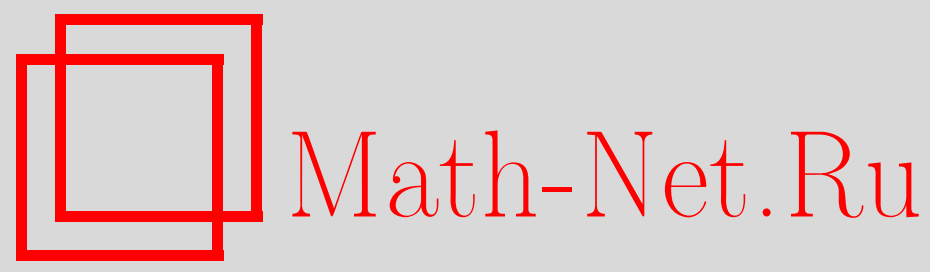

Р. Э. Яворский, Предикатные логики выразительно сильных теорий, Матем. заметки, 1999, том 66, выпуск 5, 777788

DOI: https://doi.org/10.4213/mzm1221

Использование Общероссийского математического портала Math-Net.Ru подразумевает, что вы прочитали и согласны с пользовательским соглашением http://www . mathnet.ru/rus/agreement

Параметры загрузки:

IP : 54.210 .77 .194

26 апреля 2023 г., 16:45:38

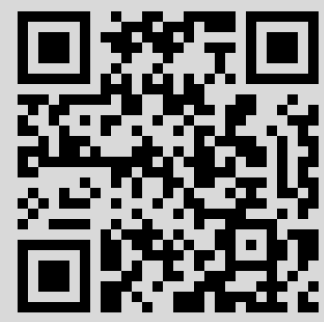




\title{
ПРЕДИКАТНЫЕ ЛОГИКИ ВЫРАЗИТЕЛЬНО СИЛЬНЫХ ТЕОРИЙ
}

\section{Р.Э. Яворский}

\begin{abstract}
В работе доказано, что для теории полей, теории колец, теории групп и всех арифметически корректных теорий логика $L(T)$ совпадает с исчислением предикатов.

Библиография: 16 названий.
\end{abstract}

1. Введение. Язык первого порядка, содержащий счетный набор предикатных символов любой валентности, без функциональных символов, констант и равенства будем назьвать предикатным языком. Через РС обозначим исчисление предикатов в этом языке. Предикатной логикой будем назьвать любую теорию в указанном языке, замкнутую относительно правила подстановки.

Пусть фиксирована теория $T$ первого порядка. Интерпретацией предикатного языка в язьке теории $T$ называется отображение $f$, сопоставляющее каждой атомарной формуле предикатного языка некоторую формулу в языке теории $T$ с тем же набором свободных переменных, причем $f$ коммутирует с булевыми связками, кванторами и операцией замены переменных. Предикатная логика $\mathcal{L}(T)$ теории $T$ есть по определению множество предикатных формул $\varphi$, таких что при любой интерпретации формула $f(\varphi)$ выводима в теории $T$.

Можно сказать, что логика $\mathcal{L}(T)$ теории $T$ описывает все логические законы, действующие в рамках теории $T$.

В работе [1] рассматривались так называемые геделевы теории, а именно те, для которых $\mathcal{L}(T)=$ РС. Было замечено, что каждая геделевская теория неразрешима, а также доказано, что арифметика Пеано РА и некоторые ее подтеории являются геделевскими теориями.

Свойства логик конструктивных арифметических теорий подробно были изучены в [2]-[4] и других работах В. Е. Плиско. В частности, им было доказано, что для любой конструктивной арифметической теории $T$ имеет место 1-сводимость теории $T$ к ее предикатной логике $\mathcal{L}(T)$ и что структура конструктивных арифметических теорий с отношением включения $\subseteq$ изоморфна структуре предикатных логик этих теорий с отношением $\subseteq$.

Предикатные логики классических арифметических теорий рассматривались в работе [5]. Было показано, что для любой степени неразрешимости $d$ существует расширение $T$ арифметики Пеано РА такое, что степени неразрешимости $T$ и $\mathcal{L}(T)$ равны $d$.

Работа выполнена при частичной поддержке Российского фонда фундаментальных исследований, гранты № 96-01-01395, № 98-01-00249. 
Определение предикатных логик теорий первого порядка является частным случаем более общего понятия, введенного в работе [6]. Пусть заданы теория $T$, язык $L$ и некоторьй класс $\mathcal{F}$ интерпретаций язька $L$ в язык теории $T$. Тогда логика $\mathcal{L}(T, L, \mathcal{F})$ есть по определению множество формул языка $L$, вьводимых в теории $T$ при любой интерпретации из класса $\mathcal{F}$. Если в качестве $L$ взять предикатный язык, а в качестве $\mathcal{F}$ - класс всех его интерпретаций в язык теории $T$, то $\mathcal{L}(T, L, \mathcal{F})$ совпадет с предикатной логикой $\mathcal{L}(T)$, которая и является объектом нашего изучения.

Данная статья продолжает цикл работ автора [7]-[9], посвященных изучению предикатных логик, соответствующих различным классическим теориям.

2. Признаки включения предикатных логик теорий. Заметим, что для произвольной теории $T$ логика $\mathcal{L}(T)$ действительно является предикатной логикой - в $\mathcal{L}(T)$ допустимо правило подстановки. $\mathrm{C}$ другой стороны, любую предикатную логику $\mathcal{L}$ можно рассматривать как логику некоторой теории первого порядка, например, себя самой. Действительно, $\mathcal{L}(\mathcal{L})=\mathcal{L}$.

Перечислим основные признаки включения предикатньх логик теорий. Пусть $\mathcal{L}_{1}$ обозначает множество предикатных формул, истинных во всех одноэлементных моделях.

ПРЕДЛОЖЕНИЕ 1. Для любой теории Т имеет место включение:

$$
\mathrm{PC} \subseteq \mathcal{L}(T) \subseteq \mathcal{L}_{1}
$$

ДоКАЗАТЕЛЬСТВо. Включение РС $\subseteq \mathcal{L}(T)$ вытекает из теоремы Гёделя о полноте классического исчисления предикатов. Докажем, что $\mathcal{L}(T) \subseteq \mathcal{L}_{1}$. Пусть $\varphi-$ предикатная формула такая, что $\varphi \notin \mathcal{L}_{1}$, и $\mathcal{M}$ - одноэлементная модель, опровергающая $\varphi$ : $\mathcal{M} \not=\varphi$. Каждый предикатньй символ является либо тождественно истинным, либо тождественно ложным в $\mathcal{M}$. Определим интерпретацию $f$ в язык теории $T$ следующим образом:

$$
f\left(R\left(x_{1}, \ldots, x_{n}\right)\right) \rightleftharpoons \begin{cases}\text { Т, } & \text { если } \mathcal{M} \models \forall a_{1} \ldots a_{n} R\left(a_{1}, \ldots, a_{n}\right), \\ \perp, & \text { если } \mathcal{M} \models \forall a_{1} \ldots a_{n} \neg R\left(a_{1}, \ldots, a_{n}\right) .\end{cases}
$$

Здесь $\top$ и $\perp-$ соответственно тождественная истина и тождественная ложь, естественным образом выразимые в любой теории первого порядка. С помощью простой индукции по построению формулы можно показать, что $\mathcal{M}=\psi \Leftrightarrow T \vdash f(\psi)$, где $\psi$ - произвольная формула. Поэтому $T \vdash \neg f(\varphi)$ и $\varphi \notin \mathcal{L}(T)$.

ПРЕДЛОЖЕНИЕ 2. Если теория $T_{2}$ является расиирением теории $T_{1}$ в том же языке, то $\mathcal{L}\left(T_{1}\right) \subseteq \mathcal{L}\left(T_{2}\right)$.

ДокАЗАТЕЛЬСТво. Поскольку язык теории $T_{1}$ совпадает с язьком теории $T_{2}$, множество всевозможных интерпретаций предикатного языка будет тем же. С другой стороны, для любой интерпретации $f$ если $T_{1} \vdash f(\varphi)$, то $T_{2} \vdash f(\varphi)$, так как $T_{2}$ есть расширение $T_{1}$. Поэтому из $\varphi \in \mathcal{L}\left(T_{1}\right)$ следует $\varphi \in \mathcal{L}\left(T_{2}\right)$.

ПРЕДЛОЖЕНИЕ 3. Если теория $T_{2}$ является консервативным расиирением теоpuи $T_{1}$, mo $\mathcal{L}\left(T_{2}\right) \subseteq \mathcal{L}\left(T_{1}\right)$. 
ДокАЗАТЕЛЬСТво. Пусть $\varphi \notin \mathcal{L}\left(T_{1}\right)$. Это означает, что $T_{1} \forall f(\varphi)$ для некоторой интерпретации $f$ в язык теории $T_{1}$. Поскольку язык $T_{2}$ расширяет язык $T_{1}$, отображение $f$ также является интерпретацией предикатного языка в язык теории $T_{2}$. В то же время, $T_{2} \not \forall f(\varphi)$ ввиду консервативности над $T_{1}$. Поэтому $\varphi \notin \mathcal{L}\left(T_{2}\right)$.

ПРЕДЛОЖЕНИЕ 4. Пусть существует интерпретация $g$ языка теории $T_{1}$ в язык теории $T_{2}$ такая, что для любой формулы $\varphi$ в языке $T_{1}$ из $T_{2} \vdash g(\varphi)$ следует $T_{1} \vdash \varphi$. Tогда $\mathcal{L}\left(T_{2}\right) \subseteq \mathcal{L}\left(T_{1}\right)$.

ДокАЗАТЕЛЬСтво. Пусть $\varphi \notin \mathcal{L}\left(T_{1}\right)$ и для интерпретации $f$ в язык теории $T_{1}$ имеет место $T_{1} \nvdash f(\varphi)$. Рассмотрим интерпретацию $h$ предикатного языка в языке теории $T_{2}$, являющуюся композицией $f$ и $g: h(\psi)=g(f(\psi))$ для любой $\psi$. По условию из $T_{1} \nvdash f(\varphi)$ следует $T_{2} \not \forall g(f(\varphi))$, т.е. $T_{2} \nvdash h(\varphi) ;$ отсюда $\varphi \notin \mathcal{L}\left(T_{2}\right)$.

ПРЕДЛОЖЕНИЕ 5. Пусть $T^{\prime}$ получена из $T$ в результате стандартной процедуры расширения с помощъю определений. Тогда $\mathcal{L}\left(T^{\prime}\right)=\mathcal{L}(T)$.

ДокАЗАТЕльство. Следует непосредственно из предыдущего признака, так как в этом случае существует каноническая интерпретация язька теории $T^{\prime}$ в язьке теории $T$ и обратно с требуемыми свойствами (см., например, [10]).

Следующее определение заимствовано из [11].

ОПРЕДЕЛЕНИЕ 1 . Пусть $\mathcal{M}_{1}$ - модель сигнатуры $\sigma_{1}=\left\langle P_{1}^{n_{1}}, \ldots, P_{k}^{n_{k}}\right\rangle$, не содержащей функциональных символов и констант, $\mathcal{M}_{2}-$ модель сигнатуры $\sigma_{2}$. Будем говорить, что модель $\mathcal{M}_{1}$ относительно әлементарно определима в модели $\mathcal{M}_{2}$, если существуют формулы $\delta(x), P_{1}^{*}\left(x_{1}, \ldots, x_{n_{1}}\right), \ldots, P_{k}^{*}\left(x_{1}, \ldots, x_{n_{k}}\right)$ сигнатуры $\sigma_{2}$ такие, что

1) множество $K \rightleftharpoons\left\{a\left|\mathcal{M}_{2}\right|=\delta(a)\right\}$ не пусто,

2 ) модель $\mathcal{K}$ сигнатуры $\sigma_{1}$, основное множество которой есть $K$, а предикаты $P_{i}$ определены формулами $P_{i}^{*}\left(x_{1}, \ldots, x_{n_{i}}\right)$, элементарно эквивалентна $\mathcal{M}_{1}$.

Пусть $\operatorname{Th}(\mathcal{M})$ обозначает элементарную теорию модели $\mathcal{M}$.

ПРЕДЛОЖЕНИЕ 6. Пусть в модели $\mathcal{M}_{1}$ выразима по крайней мере одна константа, и $\mathcal{M}_{1}$ относительно әлементарно определима в $\mathcal{M}_{2}$. Тогда $\mathcal{L}\left(\operatorname{Th}\left(\mathcal{M}_{2}\right)\right) \subseteq$ $\mathcal{L}\left(\operatorname{Th}\left(\mathcal{M}_{1}\right)\right)$.

ДокАЗАТЕльство. Воспользуемся предложением 4. Для этого построим подходящую интерпретацию $g$ языка $\sigma_{1}$ в язык $\sigma_{2}$. Пусть $C(x)$ - формула, определяющая в $\mathcal{M}_{1}$ некоторую константу. Тогда $\mathcal{M}_{1} \models \exists ! x C(x)$ ( $\exists ! x$ обозначает “существует единственное $x "$ ) и, следовательно, $\mathcal{K}=\exists ! x C(x)$, поскольку модели $\mathcal{K}$ и $\mathcal{M}_{1}$ элементарно эквивалентны. Обозначим через $c$ тот элемент множества $K$, для которого $\mathcal{K} \models C(c)$. Рассмотрим отношение эквивалентности на $\mathcal{M}_{2}$, заданное формулой

$$
x \approx y \rightleftharpoons(x=y) \vee(\neg \delta(x) \& y=c) \vee(\neg \delta(y) \& x=c) \vee(\neg \delta(x) \& \neg \delta(y))
$$

Это отношение совпадает с равенством на элементах множества $K$, а все остальные элементы из $\left|\mathcal{M}_{2}\right|$ (носителя модели $\mathcal{M}_{2}$ ), не попадающие в $K$, полагает эквивалентньми константе $c$. 
Определим теперь интерпретацию $g$ следующим образом: для любого предикатного символа $P$ сигнатуры $\sigma_{1}$

$$
\begin{aligned}
g\left(P\left(x_{1}, \ldots, x_{k}\right)\right) \rightleftharpoons & \exists z_{1}, \ldots, z_{k}\left(\delta\left(z_{1}\right) \& \ldots \& \delta\left(z_{k}\right)\right. \\
& \left.\& x_{1} \approx z_{1} \& \ldots \& x_{k} \approx z_{k} \& P^{*}\left(z_{1}, \ldots, z_{k}\right)\right) .
\end{aligned}
$$

Пусть $A\left(x_{1}, \ldots, x_{n}\right)$ - формула сигнатуры $\sigma_{1}, a_{1}, \ldots, a_{n}$-произвольные элементы $K$. Обозначим через $A^{g}\left(a_{1}, \ldots, a_{n}\right)$ результат подстановки $a_{1}, \ldots, a_{n}$ в формулу $g\left(A\left(x_{1}\right.\right.$, $\left.\left.\ldots, x_{n}\right)\right)$. Индукцией по построению формулы $A$ докажем, что для любых $a_{1}, \ldots, a_{n}$ имеет место соотношение:

$$
\mathcal{K} \models A\left(a_{1}, \ldots, a_{n}\right) \Leftrightarrow \mathcal{M}_{2} \models A^{g}\left(a_{1}, \ldots, a_{n}\right) .
$$

Если $A$ совпадает с одним из $P_{i}$, это следует из определений $g$ и $\mathcal{K}$. Действительно, имеет место следующая цепочка равносильностей:

$$
\begin{aligned}
\mathcal{K} & \models P_{i}\left(a_{1}, \ldots, a_{n_{i}}\right) \Leftrightarrow \mathcal{M}_{2} \models P_{i}^{*}\left(a_{1}, \ldots, a_{n_{i}}\right) \\
& \Leftrightarrow \mathcal{M}_{2}=\exists z_{1} \ldots z_{n_{i}}\left(\bigwedge_{1 \leqslant j \leqslant n_{i}} a_{j}=z_{j} \& P_{i}^{*}\left(z_{1}, \ldots, z_{n_{i}}\right)\right) .
\end{aligned}
$$

Поскольку $a_{j}=z_{j}$ эквивалентно $\delta\left(z_{j}\right) \& z_{j} \approx a_{j}$ для элементов $a_{j} \in K$, имеем

$$
\begin{aligned}
& \mathcal{M}_{2} \models \exists z_{1} \ldots z_{n_{i}}\left(\bigwedge_{1 \leqslant j \leqslant n_{i}} \delta\left(z_{j}\right) \& \bigwedge_{1 \leqslant j \leqslant n_{i}} a_{j} \approx z_{j} \& P_{i}^{*}\left(z_{1}, \ldots, z_{n_{i}}\right)\right) \\
& \Leftrightarrow \mathcal{M}_{2} \models P^{g}\left(a_{1}, \ldots, a_{n}\right) .
\end{aligned}
$$

Шаг индукции в случае, когда главной в $A$ является одна из булевых связок, тривиален. Пусть $A$ имеет вид $\exists y B\left(x_{1}, \ldots, x_{n}, y\right)$ и $\mathcal{K} \models \exists y B\left(a_{1}, \ldots, a_{n}, y\right)$. Тогда найдется элемент $b \in K$ такой, что $\mathcal{K} \models B\left(a_{1}, \ldots, a_{n}, b\right)$, что по предположению индукции равносильно $\mathcal{M}_{2}=B^{g}\left(a_{1}, \ldots, a_{n}, b\right)$. Отсюда следует, что $\mathcal{M}_{2} \models \exists y B^{g}\left(a_{1}, \ldots, a_{n}, y\right)$, т.е. $\mathcal{M}_{2}=A^{g}\left(a_{1}, \ldots, a_{n}\right)$.

Покажем, что верно и обратное. Пусть $\mathcal{M}_{2} \models \exists y B^{g}\left(a_{1}, \ldots, a_{n}, y\right)$ и $d$ - элемент из $\left|\mathcal{M}_{2}\right|$ такой, что $\mathcal{M}_{2} \models B^{g}\left(a_{1}, \ldots, a_{n}, d\right)$. Если $d \in K$, то по предположению индукции $\mathcal{K} \models B\left(a_{1}, \ldots, a_{n}, d\right)$, откуда $\mathcal{K} \models \exists y B\left(a_{1}, \ldots, a_{n}, y\right)$, что и требовалось доказать. В случае, если $d \notin K$, можно показать, что $\mathcal{M}_{2} \models B^{g}\left(a_{1}, \ldots, a_{n}, c\right)$. Действительно, непосредственно из определения $g$ следует, что для любой формулы $D\left(x_{1}, \ldots, x_{k}\right)$ сигнатуры $\sigma_{1}$ имеет место соотношение:

$$
\mathcal{M}_{2} \models y_{1} \approx y_{2} \rightarrow\left(D^{g}\left(x_{1}, \ldots, x_{k-1}, y_{1}\right) \leftrightarrow D^{g}\left(x_{1}, \ldots, x_{k-1}, y_{2}\right)\right) .
$$

Поскольку $d \notin K$, то $d \approx c$. Следовательно,

$$
\mathcal{M}_{2} \models B^{g}\left(a_{1}, \ldots, a_{n}, c\right)
$$

откуда $\mathcal{K} \models B\left(a_{1}, \ldots, a_{n}, c\right)$ и $\mathcal{K} \models \exists y B\left(a_{1}, \ldots, a_{n}, y\right)$, что и требовалось доказать.

Итак, для любой замкнутой формулы $\varphi$ сигнатуры $\sigma_{1}$ вьполняется

$$
\mathcal{K} \models \varphi \Leftrightarrow \mathcal{M}_{2}=g(\varphi)
$$

Поскольку модели $\mathcal{K}$ и $\mathcal{M}_{1}$ элементарно эквивалентны, отсюда следует, что $\mathcal{M}_{1} \models$ $\varphi \Leftrightarrow \mathcal{M}_{2} \mid=g(\varphi)$.

В силу предложения 4 получаем $\mathcal{L}\left(\operatorname{Th}\left(\mathcal{M}_{2}\right)\right) \subseteq \mathcal{L}\left(\operatorname{Th}\left(\mathcal{M}_{1}\right)\right)$. 
3. Предикатные логики для выразительно сильных теорий. Покажем теперь, что для теории колец, теории полей, теории групп и всех арифметически корректных теорий логика совпадает с исчислением предикатов РС. Ниже под предикатной логикой модели мы подразумеваем предикатную логику элементарной теории этой модели.

В этой главе неоднократно будет использовано следующее простое соображение.

ЗАмЕчАниЕ 1. Ввиду того, что включение $\mathrm{PC} \subseteq \mathcal{L}(T)$ имеет место для любой классической теории, для доказательства равенства $\mathcal{L}(T)=\mathrm{PC}$ достаточно показать, что $\mathcal{L}(T) \subseteq \mathrm{PC}$.

3.1. Арифметически корректные теории. Будем говорить, что теория $T$ aрuфметически корректна, если в ней выразимы все арифметические отношения, и ее теоремы истинны в стандартной модели арифметики.

ТЕОрема 1. Для любой арифметически корректной теории $T$ логика $\mathcal{L}(T)$ совпадает с исчислением предикатов РС.

ДоказАтельство. Покажем, что $\mathcal{L}(T) \subseteq$ РС. Пусть $\varphi \notin$ РС, т.е. $\neg \varphi$ выполнима. Как следует из результатов о формализуемости теоремы о полноте логики предикатов в арифметике [12], [13], всякая вьполнимая формула вьполнима также и в области натуральных чисел, а именно, найдутся арифметические отношения

$$
P_{1}^{A}\left(x_{1}, \ldots, x_{k_{1}}\right), \ldots, P_{n}^{A}\left(x_{1}, \ldots, x_{k_{n}}\right), \ldots
$$

такие, что если предикатные символы

$$
P_{1}\left(x_{1}, \ldots, x_{k_{1}}\right), \ldots, P_{n}\left(x_{1}, \ldots, x_{k_{n}}\right), \ldots
$$

интерпретировать соответственно этими отношениями, то формула $\neg \varphi$ будет истинной в стандартной модели арифметики.

Обозначим через $A_{i}\left(x_{1}, \ldots, x_{k_{i}}\right)$ формулу в языке теории $T$, выражающую отношение $P_{i}^{A}\left(x_{1}, \ldots, x_{k_{i}}\right)$. Определим интерпретацию $f$ следующим образом:

$$
f\left(P_{i}\left(x_{1}, \ldots, x_{k_{i}}\right)\right) \rightleftharpoons A_{i}\left(x_{1}, \ldots, x_{k_{i}}\right)
$$

При такой интерпретации формула $f(\varphi)$ будет ложной в стандартной модели арифметики, поэтому $T \nvdash f(\varphi)$ и $\varphi \notin \mathcal{L}(T)$.

Из этой теоремы получаем:

СлЕДСТВИЕ 1. Исчисление предикатов $\mathrm{PC}$ является логикой истинностной арифметики ТА и всех ее подтеорий в полном арифметическом языке, в частности, арифметики Пеано РА.

\section{2. Теория колец.}

ТЕорема 2. а) Логика әлементарной теории кольца иелых чисел совпадает с исчислением предикатов РС.

б) Логика теории колеи, совпадает с РС. 
ДокАЗАТЕЛЬСтво. а) Пусть ТА обозначает элементарную теорию стандартной модели арифметики $\langle\mathbb{N}, x+y=z, x \cdot y=z\rangle$ в языке, содержащем два предикатных символа для операций сложения и умножения: $x+y=z$ и $x \cdot y=z$. Воспользуемся предложением 6. Заметим, что в ТА выразимы все константы для натуральных чисел. Покажем, что $\langle\mathbb{N}, x+y=z, x \cdot y=z\rangle$ относительно элементарно определима в кольце цельх чисел $\langle\mathbb{Z},+, \cdot\rangle$. Действительно, поскольку любое натуральное число представимо в виде суммы четырех квадратов целых чисел [15], следующие формулы

$$
\begin{gathered}
\delta(x) \rightleftharpoons \exists y u v w(x=y \cdot y+u \cdot u+v \cdot v+w \cdot w), \\
(x+y=z)^{*} \rightleftharpoons x+y=z, \\
(x \cdot y=z)^{*} \rightleftharpoons x \cdot y=z
\end{gathered}
$$

очевидньм образом выделяют в $\langle\mathbb{Z},+, \cdot\rangle$ модель, изоморфную стандартной модели арифметики. В силу предложения $6 \mathcal{L}(\langle\mathbb{Z},+, \cdot\rangle) \subseteq \mathcal{L}(\mathrm{TA})$. Согласно следствию 1 и замечанию 1 имеем $\mathcal{L}(\langle\mathbb{Z},+, \cdot\rangle)=\mathrm{PC}$.

б) Пусть $\mathbf{K}$ обозначает элементарную теорию класса всех колец. Поскольку $\operatorname{Th}\langle\mathbb{Z},+, \cdot\rangle$ является расширением $\mathbf{K}$ в том же язьке, в силу предложения 2 получаем $\mathcal{L}(\mathbf{K}) \subseteq \mathcal{L}(\mathrm{Th}\langle Z,+, \cdot\rangle)$, откуда $\mathcal{L}(\mathbf{K})=$ PC.

Теорема доказана.

\section{3. Теория полей.}

Теорема 3. а) Логика элементарной теории поля рациональных чисел совпадает с исчислением предикатов РС.

б) Логика теории полей совпадает $c$ PC.

ДокАЗАтЕЛьство. а) Мы воспользуемся известным результатом Дж. Робинсон о том, что понятия целого и натурального числа определимы в терминах понятия рационального числа и операций сложения и умножения над ними. В [14] доказано (теорема 3.1.i), что для того, чтобы рациональное число $u$ было целым, необходимо и достаточно вьполнение следующей формулы [14]:

$$
\begin{aligned}
& \forall a b\left(\left(\exists x y z ( 2 + b z ^ { 2 } = x ^ { 2 } + a y ^ { 2 } ) \wedge \forall m \left(\exists x y z \left(2+a b m^{2}+b z^{2}\right.\right.\right.\right. \\
& \left.\left.\left.\quad=x^{2}+a y^{2}\right) \rightarrow \exists x y z\left(2+a b(m+1)^{2}+b z^{2}=x^{2}+a y^{2}\right)\right)\right) \\
& \left.\quad \rightarrow \exists x y z\left(2+a b u^{2}+b z^{2}=x^{2}+a y^{2}\right)\right) .
\end{aligned}
$$

Если обозначить ее через $\operatorname{Int}(u)$, то для выражения понятия натурального числа можно использовать следующую формулу:

$$
\delta(u) \rightleftharpoons \operatorname{Int}(u) \wedge u \neq 0 \wedge \exists x y\left(\operatorname{Int}(x) \wedge x \neq 0 \wedge\left(u=x^{2} \vee x^{2}=1+u y^{2}\right)\right) .
$$

Таким образом, стандартная модель арифметики относительно элементарно определима в поле рациональных чисел. Согласно предложению 6 получаем включение $\mathcal{L}(\langle Q,+, \cdot\rangle) \subseteq \mathcal{L}(\mathrm{TA})$. Так как $\mathcal{L}(\mathrm{TA})=$ РС, согласно замечанию 1 получаем равенство $\mathcal{L}(\langle Q,+, \cdot\rangle)=$ PC.

б) Пусть $\mathbf{F}$ обозначает элементарную теорию класса всех полей. В силу предложения 2 имеет место включение $\mathcal{L}(\mathbf{F}) \subseteq \mathcal{L}(\langle Q,+, \cdot\rangle)$. Отсюда $\mathcal{L}(\mathbf{F})=\mathrm{PC}$.

Теорема доказана. 
3.4. Теория групп. Пусть $\mathbf{G}$ обозначает элементарную теорию групп в языке, содержащем единственную нелогическую константу - бинарный функциональньй символ.

Пусть $R$ - кольцо с единицей. Квадратная матрица над кольцом $R$ назьвается верxней унитреугольной, если все ее элементы под главной диагональю равны нулю и все ее элементы на главной диагонали равны 1 . Если кольцо $R$ ассоциативно, то множество всех верхних унитреугольных матриц над $R$ образует группу относительно матричного умножения, которая обозначается $U T_{n}(R)$.

ТЕОрема 4. а) Логика әлементарной теории группы $U T_{3}(\mathbb{Z})$ верхних унитреугольных $3 \times 3$ матрии над кольиом челых чисел совпадает с исчислением предикатов $\mathrm{PC}$.

б) Логика теории групп совпадает с исчислением предикатов РС.

ДокАЗАТЕЛьСтво. а) План доказательства теоремы следующий. Мы рассмотрим две вспомогательные модели: модель

$$
\mathcal{M}_{1}=\langle\mathbb{N}, P(x, y, z), x \cdot y=z\rangle
$$

где $P(x, y, z)$ обозначает предикат $x+y=z \vee|x-y|=z$, и модель

$$
\mathcal{M}_{2}=\langle\mathbb{Z}, x+y=z,|x \cdot y|=|z|\rangle
$$

и докажем, что $\mathcal{L}\left(\mathcal{M}_{1}\right)=\mathrm{PC}, \mathcal{L}\left(\mathcal{M}_{2}\right) \subseteq \mathcal{L}\left(\mathcal{M}_{1}\right)$ и $\mathcal{L}\left(U T_{3}(\mathbb{Z})\right) \subseteq \mathcal{L}\left(\mathcal{M}_{2}\right)$.

ЛЕмма 1. В модели $\mathcal{M}_{1}$ выразим предикат сложения.

ДокАЗАТЕЛЬСтво. Заметим, что формула

$$
\begin{aligned}
\psi\left(y, x_{1}, x_{2}\right) \rightleftharpoons & \exists u, v\left(P\left(x_{1}, x_{2}, u\right) \& P\left(x_{1}, x_{2}, v\right) \& y=u \cdot v\right. \\
& \left.\&\left(x_{1} \neq 0 \& x_{2} \neq 0 \rightarrow u \neq v\right)\right)
\end{aligned}
$$

выражает отношение $y=\left|x_{1}^{2}-x_{2}^{2}\right|$ в $\mathcal{M}_{1}$.

Покажем, что для любого $n \geqslant 1$ в модели $\mathcal{M}_{1}$ выразимо отношение $y=x_{1}^{2}+\cdots+x_{n}^{2}$. Индукция по $n$. Для $n=1$ это тривиально:

$$
y=x_{1}^{2} \rightleftharpoons y=x_{1} \cdot x_{1}
$$

Шаг индукции. Пусть отношение $y=x_{1}^{2}+\cdots+x_{k}^{2}$ выразимо. Для того чтобы выразить отношение $y=x_{1}^{2}+\cdots+x_{k}^{2}+x_{k+1}^{2}$, рассмотрим формулу:

$$
\begin{aligned}
& \varphi\left(y, x_{1}, \ldots, x_{k+1}\right) \rightleftharpoons\left(x_{1}=0 \& y=x_{2}^{2}+\cdots+x_{k+1}^{2}\right) \vee \cdots \\
& \quad \vee\left(x_{k+1}=0 \& y=x_{1}^{2}+\cdots+x_{k}^{2}\right) \\
& \quad \vee\left(x_{1} \neq 0 \& \cdots \& x_{k+1} \neq 0\right. \\
& \quad \& \exists u, v\left(u=x_{1}^{2}+\cdots+x_{k}^{2} \& v=x_{k+1}^{2} \& P(u, v, y)\right. \\
& \left.\quad \& \forall w\left(\exists z\left(z=x_{1}^{2}+\cdots+x_{k-1}^{2} \& P(y, z, w)\right) \rightarrow w \neq\left|x_{k}^{2}-x_{k+1}^{2}\right|\right)\right)
\end{aligned}
$$


Отметим, что при $k=1$ условие $z=a_{1}^{2}+\cdots+a_{k-1}^{2}$ преврашается в $z=0$, в результате чего импликация в последнем конъюнктивном члене приобретает вид

$$
\forall w(P(b, 0, w)) \rightarrow w \neq\left|a_{1}^{2}-a_{2}^{2}\right|
$$

т.е. $b \neq\left|a_{1}^{2}-a_{2}^{2}\right|$.

Покажем, что для любых натуральных чисел $b, a_{1}, \ldots, a_{k+1}$ имеет место эквивалентность

$$
\mathcal{M}_{1}=\varphi\left(b, a_{1}, \ldots, a_{k+1}\right) \Leftrightarrow b=a_{1}^{2}+\cdots+a_{k+1}^{2} .
$$

Действительно, если хотя бы одно из $a_{i}$ равно нулю, эта эквивалентность следует из предположения индукции о выразимости отношения $y=x_{1}^{2}+\cdots+x_{k}^{2}$. Рассмотрим теперь случай, когда все $a_{i}$ отличны от нуля.

Докажем импликацию слева направо. Пусть $\mathcal{M}_{1}=\varphi\left(b, a_{1}, \ldots, a_{k+1}\right)$. Так как все $a_{i}$ не равны нулю, истинным является последний член дизъюнкции, а именно:

$$
\begin{aligned}
\mathcal{M}_{1}= & \exists u, v\left(u=a_{1}^{2}+\cdots+a_{k}^{2} \& v=a_{k+1}^{2} \& P(u, v, b)\right. \\
& \left.\& \forall w\left(\exists z\left(z=a_{1}^{2}+\cdots+a_{k-1}^{2} \& P(b, z, w)\right) \rightarrow w \neq\left|a_{k}^{2}-a_{k+1}^{2}\right|\right)\right) .
\end{aligned}
$$

Из истинности первого члена конъюнкции следует, что

$$
b=a_{1}^{2}+\cdots+a_{k+1}^{2} \text { либо } b=\left|a_{1}^{2}+\cdots+a_{k}^{2}-a_{k+1}^{2}\right| .
$$

Предположим, что $b=\left|a_{1}^{2}+\cdots+a_{k}^{2}-a_{k+1}^{2}\right|$. Покажем, что в этом случае нарушается последний конъюнктивный член. Действительно, посылка импликации будет истинной, если

$$
w=b+a_{1}^{2}+\cdots+a_{k-1}^{2} \text { или } w=\left|b-\left(a_{1}^{2}+\cdots+a_{k-1}^{2}\right)\right| .
$$

Подставив значение $b$, получим, что

$$
\begin{aligned}
& w=\left|a_{1}^{2}+\cdots+a_{k}^{2}-a_{k+1}^{2}\right|+a_{1}^{2}+\cdots+a_{k-1}^{2} \text { или } \\
& w=|| a_{1}^{2}+\cdots+a_{k}^{2}-a_{k+1}^{2}\left|-\left(a_{1}^{2}+\cdots+a_{k-1}^{2}\right)\right| .
\end{aligned}
$$

Независимо от знака суммы $a_{1}^{2}+\cdots+a_{k}^{2}-a_{k+1}^{2}$ после раскрытия модулей получим, что одно из значений $w$ будет равно

$$
w=\left|a_{1}^{2}+\cdots+a_{k-1}^{2}-\left(a_{1}^{2}+\cdots+a_{k+1}^{2}\right)\right|=\left|a_{k}^{2}-a_{k+1}^{2}\right|,
$$

что означает ложность импликации.

Докажем теперь импликацию справа налево в (*). Как уже было замечено, достаточно рассмотреть случай, когда все $a_{i}$ отличны от нуля. Итак, пусть $b=a_{1}^{2}+\cdots+$ $a_{k+1}^{2}$. Покажем, что в этом случае будет истинным последний дизъюнктивный член в $\varphi\left(b, a_{1}, \ldots, a_{k+1}\right)$. Истинность первого конъюнктивного члена в нем следует из истинности $P\left(a_{1}^{2}+\cdots+a_{k}^{2}, a_{k+1}^{2}, b\right)$.

Покажем, что будет истинным второй конъюнктивный член:

$$
\mathcal{M}_{1} \models \forall w\left(\exists z\left(z=a_{1}^{2}+\cdots+a_{k-1}^{2} \& P(b, z, w)\right) \rightarrow w \neq\left|a_{k}^{2}-a_{k+1}^{2}\right|\right) .
$$


Действительно, посылка импликации будет истинной, если

$$
\begin{aligned}
& w=b+a_{1}^{2}+\cdots+a_{k-1}^{2}=2 a_{1}^{2}+\cdots+2 a_{k-1}^{2}+a_{k}^{2}+a_{k+1}^{2} \text { или } \\
& w=\left|b-\left(a_{1}^{2}+\cdots+a_{k-1}^{2}\right)\right|=a_{k}^{2}+a_{k+1}^{2},
\end{aligned}
$$

а тогда будет истинньм и заключение. В обоих случаях $w \neq\left|a_{k}^{2}-a_{k+1}^{2}\right|$, поскольку $a_{k} \neq 0$ и $a_{k+1} \neq 0$.

Таким образом, соотношение $(*)$ доказано.

Для завершения доказательства леммы воспользуемся известным фактом из теории чисел о том, что любое натуральное число может быть представлено в виде суммы четырех квадратов целых чисел [15]. Исходя из этого, операцию сложения можно выразить следуюшим способом:

$$
x+y=z \rightleftharpoons \exists u_{1} \ldots u_{8}\left(x=u_{1}^{2}+\cdots u_{4}^{2} \& y=u_{5}^{2}+\cdots+u_{8}^{2} \& z=u_{1}^{2}+\cdots+u_{8}^{2}\right) .
$$

Лемма доказана.

Из этой леммы, в частности, следует, что в модели $\mathcal{M}_{1}$ выразимы все арифметические отношения. Таким образом, элементарная теория модели $\mathcal{M}_{1}$ является арифметически корректной в смысле условий теоремы 1 . Следовательно, $\mathcal{L}\left(\mathcal{M}_{1}\right)=\mathrm{PC}$.

Рассмотрим теперь модель $\mathcal{M}_{2}=\langle\mathbb{Z}, x+y=z,|x \cdot y|=|z|\rangle$.

Лемма 2. Существует интерпретаиия $f$ языка модели $\mathcal{M}_{1}$ в язык модели $\mathcal{M}_{2}$ такая, что для любой замкнутой формуль $\varphi$ в языке $\mathcal{M}_{1}$ имеет место: $\mathcal{M}_{1} \models$ $\varphi \Leftrightarrow \mathcal{M}_{2} \mid=f(\varphi)$.

ДоказАтельство. Сначала заметим, что отношение $|x|=|y|$ выразимо в $\mathcal{M}_{2}$, например, формулой $\forall u(|x u|=|y u|)$. Определим интерпретацию $f$ следующим образом:

$$
\begin{aligned}
f(x=y) & \rightleftharpoons|x|=|y|, \\
f(P(x, y, z)) & \rightleftharpoons|x+y|=|z| \vee|x-y|=|z|, \\
f(x \cdot y=z) & \rightleftharpoons|x \cdot y|=|z| .
\end{aligned}
$$

Пусть $A\left(x_{1}, \ldots, x_{n}\right)$ - произвольная формула со свободными переменными $x_{1}$, $\ldots, x_{n}$ в языке $\mathcal{M}_{1}$. Покажем, что для любого набора целых чисел $a_{1}, \ldots, a_{n}$ имеет место соотношение

$$
\mathcal{M}_{1} \models A\left(\left|a_{1}\right|, \ldots,\left|a_{n}\right|\right) \Leftrightarrow \mathcal{M}_{2} \models f\left(A\left(a_{1}, \ldots, a_{n}\right)\right),
$$

где под $f\left(A\left(a_{1}, \ldots, a_{n}\right)\right)$ мы понимаем результат подстановки $a_{1}, \ldots, a_{n}$ в формулу $f\left(A\left(x_{1}, \ldots, x_{n}\right)\right)$.

Для атомарных формул это соотношение приобретает вид:

$$
\begin{aligned}
\left|a_{1}\right| & =\left|a_{2}\right| \Leftrightarrow\left|a_{1}\right|=\left|a_{2}\right|, \\
\left|a_{1}\right|+\left|a_{2}\right|=\left|a_{3}\right| \vee|| a_{1}|-| a_{2}|| & =\left|a_{3}\right| \Leftrightarrow\left|a_{1}+a_{2}\right|=\left|a_{3}\right| \vee\left|a_{1}-a_{2}\right|=\left|a_{3}\right|, \\
\left|a_{1}\right| \cdot\left|a_{2}\right| & =\left|a_{3}\right| \Leftrightarrow\left|a_{1}\right| \cdot\left|a_{2}\right|=\left|a_{3}\right|
\end{aligned}
$$

и не требует доказательства. Индукцией по построению покажем, что это соотношение имеет место для любой формулы. 
Если формула $A$ получена применением булевых связок, шаг индукции тривиален. Пусть $A$ имеет вид $\exists y B\left(x_{1}, \ldots, x_{n}, y\right)$ и $a_{1}, \ldots, a_{n}$ - произвольньй набор целых чисел. Тогда $\mathcal{M}_{1}=\exists y B\left(\left|a_{1}\right|, \ldots,\left|a_{n}\right|, y\right)$ равносильно тому, что для некоторого натурального числа $b \mathcal{M}_{1}=B\left(\left|a_{1}\right|, \ldots,\left|a_{n}\right|, b\right)$. По предположению индукции это равносильно тому, что $\mathcal{M}_{2} \models f\left(B\left(a_{1}, \ldots, a_{n}, b\right)\right)$ и $\mathcal{M}_{2} \models f\left(B\left(a_{1}, \ldots, a_{n},-b\right)\right)$. Ввиду произвольности выбора $b$ это равносильно $\mathcal{M}_{2} \models \exists y f\left(B\left(a_{1}, \ldots, a_{n}, y\right)\right)$ и $\mathcal{M}_{2} \models f\left(A\left(a_{1}, \ldots, a_{n}\right)\right)$, поскольку $f$ коммутирует с квантором.

Лемма доказана.

Согласно предложению 4 имеем $\mathcal{L}\left(\mathcal{M}_{2}\right) \subseteq \mathcal{L}\left(\mathcal{M}_{1}\right)$.

ЛЕмма 3. Модель $\mathcal{M}_{2}=\langle\mathbb{Z}, x+y=z,|x \cdot y|=|z|\rangle$ относительно әлементарно определима в әруппе $U T_{3}(\mathbb{Z})$.

ДокАЗАТЕЛЬСтво. Этот факт непосредственно следует из результатов, изложенных в [16], и его доказательство было любезно предоставлено профессором О.В. Белеградеком.

Напомним необходимые определения. Пусть задана группа $G$. Kоммутатором $[a, b]$ элементов $a$ и $b$ назьвается элемент $a b a^{-1} b^{-1}$. Централизатором $C(a)$ элемента $a$ группы $G$ называется множество элементов группы, коммутируюших с $a$. Известно, что $C(a)$ является подгруппой в $G$. Центром $\mathrm{Z}(\mathrm{G})$ группы $G$ назьвается множество элементов, перестановочньх со всеми ее элементами.

ОПРЕДЕЛЕНИЕ 2. Пусть $R$ - коммутативное ассоциативное кольцо с единищей. Пара элементов $\left(a, a^{\prime}\right)$ назьвается базой в групе $U T_{3}(R)$, если

1) подгруппы $C(a), C\left(a^{\prime}\right)$ абелевы,

2) $C(a) \cap C\left(a^{\prime}\right)=Z\left(U T_{3}(R)\right)$,

3) $\left[a, C\left(a^{\prime}\right)\right]=\left[C(a), a^{\prime}\right]=Z\left(U T_{3}(R)\right)$.

Рассмотрим следующие формулы:

$$
\begin{aligned}
\varphi_{1}(a) & \rightleftharpoons \forall x y(a x=x a \& a y=y a \rightarrow x y=y x), \\
\varphi_{2}\left(a, a^{\prime}\right) & \rightleftharpoons \forall x\left(a x=x a \& a^{\prime} x=x a^{\prime} \leftrightarrow \forall y(x y=y x)\right), \\
\varphi_{3}^{1}\left(a, a^{\prime}\right) & \rightleftharpoons \forall x\left(\exists y\left(a^{\prime} y=y a^{\prime} \& x=a y a^{-1} y^{-1}\right) \leftrightarrow \forall y(x y=y x)\right), \\
\varphi_{3}^{2}\left(a, a^{\prime}\right) & \rightleftharpoons \forall x\left(\exists y\left(a y=y a \& x=y a^{\prime} y^{-1} a^{\prime-1}\right) \leftrightarrow \forall y(x y=y x)\right) .
\end{aligned}
$$

Как можно увидеть, формула

$$
\operatorname{Basa}\left(a, a^{\prime}\right) \rightleftharpoons \varphi_{1}(a) \& \varphi_{1}\left(a^{\prime}\right) \& \varphi_{2}\left(a, a^{\prime}\right) \& \varphi_{3}^{1}\left(a, a^{\prime}\right) \& \varphi_{3}^{2}\left(a, a^{\prime}\right)
$$

формализует понятие базы в языке теории груп, а именно, пара $\left(a, a^{\prime}\right)$ является базой, если и только если $U T_{3}(R) \models \operatorname{Basa}\left(a, a^{\prime}\right)$.

Для краткости обозначим через $(\alpha, \beta, \gamma)$ матрицу из $U T_{3}(R)$ с элементами $\alpha, \beta, \gamma$ на местах $(1,2),(2,3),(1,3)$ соответственно.

Непосредственное вычисление показывает, что

$$
\left[(\alpha, \beta, \gamma),\left(\alpha^{\prime}, \beta^{\prime}, \gamma^{\prime}\right)\right]=\left(0,0, \alpha \beta^{\prime}-\alpha^{\prime} \beta\right)
$$

$\mathrm{B}[16]$ доказано (предложение 1.1.15.7), что для $a=(\alpha, \beta, \gamma), a^{\prime}=\left(\alpha^{\prime}, \beta^{\prime}, \gamma^{\prime}\right)$ пара $\left(a, a^{\prime}\right)$ является базой, если и только если элемент $\Delta=\alpha \beta^{\prime}-\alpha^{\prime} \beta$ обратим в кольце $R$. 
В частности, для $R=\mathbb{Z}$ пара $\left(a, a^{\prime}\right)$ является базой, если и только если $\Delta=\alpha \beta^{\prime}-$ $\alpha^{\prime} \beta= \pm 1$.

В группе $U T_{3}(\mathbb{Z})$ центр состоит из матриц вида $(0,0, \gamma)$. Если $c=(0,0, \gamma), c^{\prime}=$ $\left(0,0, \gamma^{\prime}\right)$, то $c c^{\prime}=\left(0,0, \gamma+\gamma^{\prime}\right)$. Таким образом, центр естественно изоморфен аддитивной группе кольца $\mathbb{Z}$.

Обозначим через $\operatorname{Mul}\left(x, x^{\prime}, y, y^{\prime}, y^{\prime \prime}\right)$ формулу

$$
\exists z z^{\prime}\left([z, x]=e \wedge\left[z, x^{\prime}\right]=y \wedge\left[z^{\prime}, x^{\prime}\right]=e \wedge\left[x, z^{\prime}\right]=y^{\prime} \wedge\left[z, z^{\prime}\right]=y^{\prime \prime}\right) .
$$

Тогда если пара $\left(a, a^{\prime}\right)$ является базой, $c=(0,0, \gamma), c^{\prime}=\left(0,0, \gamma^{\prime}\right), c^{\prime \prime}=\left(0,0, \gamma^{\prime \prime}\right)$, то $U T_{3}(R) \models \operatorname{Mul}\left(a, a^{\prime}, c, c^{\prime}, c^{\prime \prime}\right)$ равносильно $R=\gamma \gamma^{\prime}=\Delta \gamma^{\prime \prime}$. (См. 1.1.16.1 в [16]).

$\mathrm{B}$ частности, $U T_{3}(\mathbb{Z}) \models \exists x x^{\prime}\left(\operatorname{Basa}\left(x, x^{\prime}\right) \& \operatorname{Mul}\left(x, x^{\prime}, c, c^{\prime}, c^{\prime \prime}\right)\right)$ равносильно $\mathbb{Z} \models \gamma$. $\gamma^{\prime}= \pm \gamma^{\prime \prime}$.

Отождествляя $\gamma \in \mathbb{Z} \mathrm{c}(0,0, \gamma) \in U T_{3}(\mathbb{Z})$, имеем интерпретацию модели $\mathcal{M}_{2}$ в группе $U T_{3}(\mathbb{Z})$ :

$$
\begin{aligned}
\delta(x) & \rightleftharpoons \forall y(x y=y x), \\
(x+y=z)^{*} & \rightleftharpoons x y=z, \\
(|x \cdot y|=|z|)^{*} & \rightleftharpoons \exists u u^{\prime}\left(\operatorname{Basa}\left(u, u^{\prime}\right) \& \operatorname{Mul}\left(u, u^{\prime}, x, y, z\right)\right),
\end{aligned}
$$

Лемма доказана.

Заметим, что в $\mathcal{M}_{2}$ выразима константа нуль: $x=0 \rightleftharpoons \forall y(x+y=y)$. Согласно предложению 6 получаем

$$
\mathcal{L}\left(U T_{3}(\mathbb{Z})\right) \subseteq \mathcal{L}(\langle\mathbb{Z}, x+y=z,|x \cdot y|=|z|\rangle),
$$

поэтому $\mathcal{L}\left(U T_{3}(\mathbb{Z})\right)=\mathrm{PC}$.

б) Поскольку элементарная теория грушы $U T_{3}(\mathbb{Z})$ является расширением теории групп $\mathbf{G}$, то $\mathcal{L}(\mathbf{G}) \subseteq \mathcal{L}\left(\operatorname{Th}\left(U T_{3}(\mathbf{Z})\right)\right)$ и, следовательно, $\mathcal{L}(\mathbf{G})=\mathrm{PC}$.

Теорема доказана.

Автор выражает глубокую благодарность своему научному руководителю професcopy С.Н. Артёмову за постановку задачи и постоянное внимание к работе, а также профессору О.В. Белеградеку за помощь при доказательстве теоремы 4.

\section{СПИСОК ЦИТИРОВАННОЙ ЛИТЕРАТУРЫ}

[1] Лифшиц В.А. Дедуктивная общезначимость и классы сведения // Записки научн. семин. ЛОМИ. 1967. Т. 4. С. 69-77.

[2] Плиско В.Е. Некоторые варианты понятия реализуемости для предикатных формул // Изв. АН СССР. Сер. матем. 1978. Т. 42. № 3. С. 636-653.

[3] Плиско В.Е. Конструктивная формализация теоремы Тенненбаума и ее применения // Матем. заметки. 1990. Т. 48. № 3. С. 108-118. 
[4] Плиско В.Е. Об арифметической сложности некоторых предикатных логик // Матем. заметки. 1992. Т. 52. №1. С. 94-104.

[5] Варданян В.А. Предикатная логика доказуемости без доказуемости // Интенсиональные логики и логическая структура теории. Тбилиси, 1988. С. 65-69.

[6] Artemov S., Montagna F. On first-order theories with provability operator // J. Symbolic Logic. 1994. V. 59. № 4. P. 1139-1153.

[7] Yavorsky R.E. Logical Schemes for First-Order Theories // Lecture Notes in Comuter Science. 1997. V. 1234. P. 410-418.

[8] Яворский Р. Э. Предикатные логики разрешимых фрагментов арифметики // Вестн. МГУ. Сер. 1 Матем. , мех. 1998. № 2. С. 12-16.

[9] Яворский Р.Э. Разрешимые логики первого порядка // Фундаментальная и прикладная математика. 1998. Т. 4. № 2. С. 733-749.

[10] Клини С. К. Введение в метаматематику. М.: ИЛ, 1957.

[11] Ершов Ю. Л. Проблемы разрешимости и конструктивные модели. М.: Наука, 1980.

[12] Hilbert D., Bernays P. Grundlagen der Mathematik. II. New York-Berlin-Heidelberg-Tokyo: Springer-Verlag, 1968.

[13] Feferman S. Arithmetization of metamathematics in a general setting // Fund. Math. 1960. V. 49. P. 35-92.

[14] Robinson J. Definability and decision problem in arithmetic // J. Symbolic Logic. 1949. V. 14. № 2. P. 98-114.

[15] Бухштаб А. А. Теория чисел. М.: Учпедгиз, 1960.

[16] Белеградек О.В. Теория моделей унитреугольных и экзистенциально замкнутых групп. Дисс. ... д.ф.-м.н. НГУ, 1995.

Математический институт РАН им. В.А. Стеклова

Поступило

17.11 .1998 